\title{
Águas Oleosas Contaminadas: Ameaças, Tratamentos e Desafios
}

Fernanda F. Roman Jose L. Diaz de Tuesta Adrián M. T. Silva Joaquim L. Faria Helder T. Gomes*

\begin{abstract}
Contaminated Oily Wastewater: Threats, Treatments, and Challenges. Contaminated oily wastewaters are a source of pollution generated by different industries. They consist of an aqueous and an oily phase, containing several other pollutants, whether they come from the production processes or are naturally associated with the constituent oils. These wastewaters have a high impact on the quality of water resources, aquatic life, air, soil, and human health. Therefore, their proper treatment is essential. Research studies are typically focused on recovering the aqueous phase, although the oil phase often has a high added value that justifies its treatment and recovery. Traditional treatments, such as physical-chemical processes or biological, are usually inefficient in their treatment. Advanced Oxidation Processes (AOPs) appear as an alternative capable of adequately treating water and eliminating pollutants from organic phases of interest to recover them.
\end{abstract}

\begin{abstract}
As águas oleosas contaminadas são uma fonte de poluição gerada por várias indústrias. São constituídas por uma fase aquosa e uma oleosa, contendo ainda outros poluentes provenientes tanto dos processos produtivos, como naturalmente associados aos óleos constituintes. Esses efluentes têm um elevado impacto sobre a qualidade dos recursos hídricos, da vida aquática, do ar, do solo e da saúde humana. É por isso essencial o seu devido tratamento. Os estudos de investigação focam-se normalmente na recuperação da fase aquosa, embora a fase oleosa possua, não raras vezes, um elevado valor acrescentado que justifica o seu tratamento e recuperação. Os tratamentos tradicionais, como os processos físico-químicos ou biológicos, são normalmente ineficientes para o seu tratamento. Os processos avançados de oxidação (Advanced Oxidation Processes, AOPs) são uma alternativa viável para o tratamento adequado de águas e eliminar poluentes das fases orgânicas de interesse com o objetivo da sua recuperação.
\end{abstract}

\begin{abstract}
1. Introdução
A procura crescente de combustíveis fósseis e de produtos à base de petróleo levou a um aumento do uso e processamento de petróleos brutos, acarretando uma crescente deposição de óleos em recursos hídricos, dando origem a um efluente denominado águas oleosas [1]. O termo água oleosa engloba todos os tipos de efluentes que contenham óleo, podendo este designar gorduras de origem animal ou vegetal, hidrocarbonetos, frações de petróleo, entre outros [2]. A principal origem das águas oleosas são as indústrias de processamento e refinamento do petróleo e as atividades associadas a essa indústria, como o transporte e o armazenamento. Outras origens relevantes incluem indústrias mecânicas e metalúrgicas, de transporte marítimo, de produção
\end{abstract}

de biodiesel e a indústria alimentar [1,3-5], o que significa que as águas oleosas podem ter uma composição muito variável. Na Tabela 1 apresentam-se alguns parâmetros com valores típicos de águas oleosas provenientes de diversas origens, dando conta da multiplicidade de características encontradas para este tipo de efluentes.

Há um crescente interesse nos estudos relacionados com as águas oleosas, que se reflete no número de publicações e citações recentes (Figura 1). Nos últimos 20 anos, o número de artigos publicados nesta área cresceu aproximadamente 50 vezes (seis artigos em 2000 versus 279 artigos em 2020) (Figura 1a), enquanto o número de citações no mesmo período cresceu mais de 1000 vezes (Figura 1b). 
Tabela 1 - Características de águas oleosas provenientes de diferentes fontes.

\begin{tabular}{|c|c|c|c|c|c|c|}
\hline Fonte & $\mathrm{pH}$ & $\begin{array}{c}\mathrm{CQO} \\
\left(\mathrm{mg} \mathrm{L}^{-1}\right)\end{array}$ & $\begin{array}{c}\mathrm{CBO}_{5} \\
\left(\mathrm{mg} \mathrm{L}^{-1}\right)\end{array}$ & $\begin{array}{c}0 \& G \\
\left(\mathrm{mg} \mathrm{L}^{-1}\right)\end{array}$ & $\begin{array}{c}\text { SST } \\
\left(\mathrm{mg} \mathrm{L}^{-1}\right)\end{array}$ & Ref. \\
\hline Fluido de corte & $8,1-8,5$ & $50300-57100$ & n.d. & $4639-5590$ & n.d. & {$[6]$} \\
\hline Água oleosa de restaurante & $6,1-8,0$ & $750-5800$ & $600-2500$ & $500-4700$ & $250-650$ & [8] \\
\hline Refinaria de óleo vegetal (girassol) & 6,32 & 4100 & 4000 & 700 & 2400 & [9] \\
\hline Indústria alimentar (atum) & 7,4 & 2178 & 910 & 641 & 4102 & [11] \\
\hline $\begin{array}{c}\text { Instalação de tratamento de água } \\
\text { (Refinaria de petróleo e indústrias } \\
\text { petroquímicas, China) }\end{array}$ & $7-9$ & $300-600$ & $150-360$ & $<50$ & $<150$ & [12] \\
\hline
\end{tabular}

CQO = carência química de oxigénio, $\mathrm{CBO}_{5}=$ carência bioquímica de oxigénio, $0 \& \mathrm{G}$ = óleo e gordura, $\mathrm{SST}$ = sólidos suspensos totais, n.d. = não disponível.

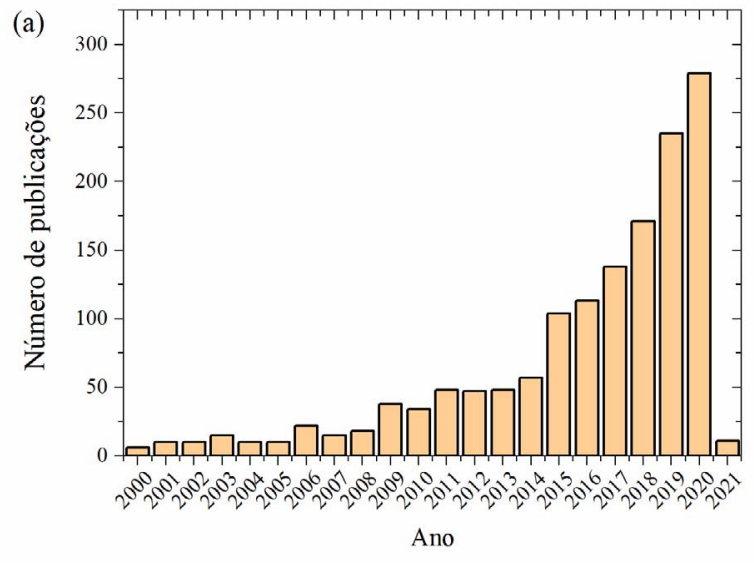

Figura 1 - (a) Número de publicações sobre águas oleosas; (b) número de citações entre 2000 e 2021. Os dados foram retirados do Web of Science em 6 de janeiro de 2021, usando a palavrachave "oily wastewater" considerando o período 2000-2021.

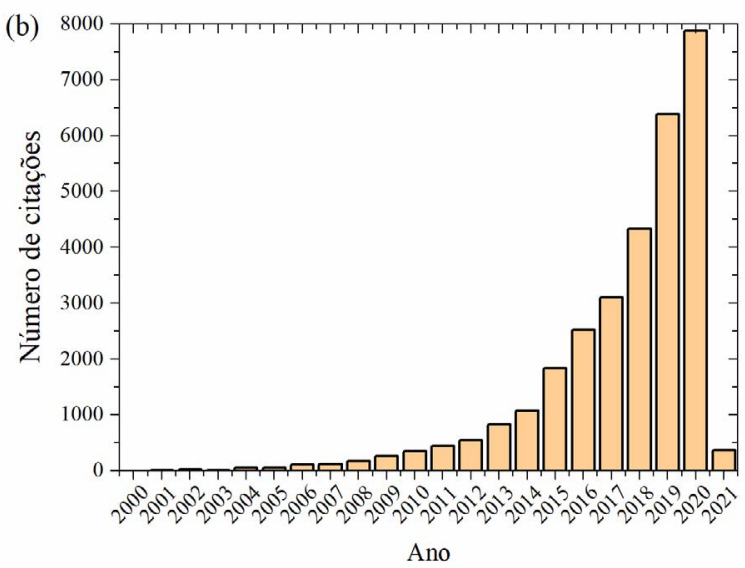

Considerando a indústria do petróleo, estima-se que, por cada tonelada de petróleo refinado, é gerada entre 0,5 e 1,0 t de águas oleosas [1], com uma concentração de óleo que pode chegar a $40 \mathrm{~g} \mathrm{~L}^{-1}$ [5], enquanto o limite máximo aceitável para a concentração de óleos e gorduras em descargas de águas residuais é de $15 \mathrm{mg} \mathrm{L}^{-1}$ (Decreto-Lei n. ${ }^{\circ}$ 236/98) [14] em Portugal. Além disso, estima-se que sejam derramados no mar a nível mundial aproximadamente 115000 barris de petróleo ( $~ 18200$ $\left.\mathrm{m}^{3}\right)$ por ano (2006-2015), representando no período indicado uma perda económica de 3,89 milhões de euros [15]. As águas oleosas podem conter também outros poluentes orgânicos e inorgânicos indesejados [3], provenientes tanto do processo produtivo, como de contaminantes que ocorrem naturalmente em petróleos brutos, como compostos sulfurados e nitrogenados. A presença desses poluentes, que podem estar presentes em ambas as fases, pode dificultar em muitos casos o processo de tratamento, nomeadamente pela formação de efluentes emulsionados. 0 descarte de águas oleosas, além de um grande problema ecológico, representa também um desperdício de recursos económicos [1-3], como a inutilização de combustíveis líquidos e produtos oleosos de elevado valor acrescentado presentes nas águas oleosas. A maior parte dos trabalhos relacionados com o tratamento de águas oleosas tem como principal objetivo apenas o tratamento da fase aquosa. No entanto, a remoção dos poluentes orgânicos, principalmente daqueles dissolvidos em fases oleosas, pode ser importante, uma vez que essa remoção permitiria a recuperação e reciclagem desta fase, evitando a extração adicional de recursos não-renováveis [3,6]. Existe, portanto, um interesse no desenvolvimento de técnicas que permitam a recuperação de fases oleosas devido aos benefícios económicos e ambientais associados [3]. 


\section{Impacto no meio ambiente e na saúde humana}

As águas oleosas contêm uma quantidade bastante elevada de compostos orgânicos, como óleo disperso e dissolvido (hidrocarbonetos, frações de petróleo, gorduras, entre outros), compostos inorgânicos dissolvidos (metais pesados, entre outros), compostos químicos inerentes ao petróleo bruto ou oriundos do processo produtivo (compostos à base de enxofre e nitrogénio e surfactantes), partículas sólidas, entre outros $[4,16]$. As águas oleosas podem afetar recursos hídricos superficiais e subterrâneos, como também influenciar a qualidade do solo e a produção agrícola, a vida marinha e a atmosfera $[4,16]$.

O óleo disperso ou solúvel é altamente tóxico e costuma estar localizado na superfície das águas [16], levando à emissão de compostos voláteis tóxicos, como hidrocarbonetos de cadeia curta. Além disso, o óleo tende a formar uma camada fina na superfície da água, diminuindo a passagem de luz e afetando o processo de fotossíntese de plantas aquáticas e a troca de oxigénio com a atmosfera. Como resultado, há uma diminuição de oxigénio dissolvido no meio aquático, afetando a vida marinha e a capacidade de autodepuração dos rios $[16,17]$. O óleo pode ainda impedir o processo de respiração de peixes bem como pode aumentar a exposição de alguns animais a condições climáticas adversas [17].

As implicações das águas oleosas não se limitam aos recursos hídricos. Afetam também as propriedades físico-químicas de solos, resultando na inibição da germinação de sementes e do crescimento de plantas. Além disso, o óleo pode ser absorvido de forma permanente pelo solo, afetando a capacidade de absorção de água e diminuindo a disponibilidade de dióxido de carbono para a respiração das plantas $[15,18]$.

No que se refere à saúde humana, o contato com águas contaminadas pode suscetibilizar o cancro de pele. O consumo de produtos de origem aquática afetados por águas contaminadas com óleo pode conduzir a problemas do trato digestivo e a danos genotóxicos [17]. Impõe-se por isso encontrar soluções efetivas para o tratamento adequado deste tipo de efluentes.

A legislação portuguesa (Decreto-Lei n. ${ }^{\circ}$ 236/98) estabelece os limites aceitáveis para águas de consumo humano [14] (Tabela 2). É possível observar que os limites de $\mathrm{CQO}, \mathrm{CBO}_{5}$ e a presença de hidrocarbonetos é significativamente inferior aos valores encontrados para uma série de águas oleosas (Tabela 1).

\section{Tratamentos convencionais}

Analogamente ao tratamento de águas residuais urbanas, o tratamento de águas oleosas combina uma série de etapas físicas, químicas e biológicas, de forma a maximizar a remoção de óleo do efluente. A Figura 2 apresenta um fluxograma simplificado das possíveis etapas para um tratamento de águas oleosas, que serão apresentadas e discutidas a seguir.

Tabela 2 - Parâmetros de qualidade de água para consumo e reutilização conforme o Decreto-Lei n. ${ }^{\circ} 236 / 98$ e o Decreto-Lei n. ${ }^{\circ} 119 / 2019$.

\begin{tabular}{|c|c|c|}
\hline Parâmetro & Valor máximo recomendável & Valor máximo admissível \\
\hline $\mathrm{pH}$ & $6,5-8,5\left(6,0-9,0^{* *}\right)$ & 9,5 \\
\hline $\mathrm{CQO}^{*}$ & $0-30 \mathrm{mg} \mathrm{L}^{-1}$ & - \\
\hline $\mathrm{CBO}_{5}^{*}$ & $3-7\left(\leq 25^{* *}\right) \mathrm{mg} \mathrm{L}^{-1}$ & $10 \mu \mathrm{g} \mathrm{L}^{-1}$ \\
\hline $\begin{array}{c}\text { Hidrocarbonetos dissolvidos } \\
\text { ou emulsionados }\end{array}$ & - & $0,2 \mu \mathrm{L} \mathrm{L}^{-1}$ \\
\hline $\begin{array}{c}\text { Hidrocarbonetos (policíclicos } \\
\text { e aromáticos) }\end{array}$ & - & - \\
\hline SST & Ausência & - \\
\hline
\end{tabular}

*Indicam valores de referência para águas superficiais destinadas a produção de água para consumo humano. ${ }^{* *}$ Indicam valores para reutilização de águas residuais tratadas em contexto de uso urbano e paisagístico.

Fonte: Decreto-Lei n. ${ }^{\circ} 236 / 98$ [14] e Decreto-Lei n. ${ }^{\circ} 119 / 2019$ [19].

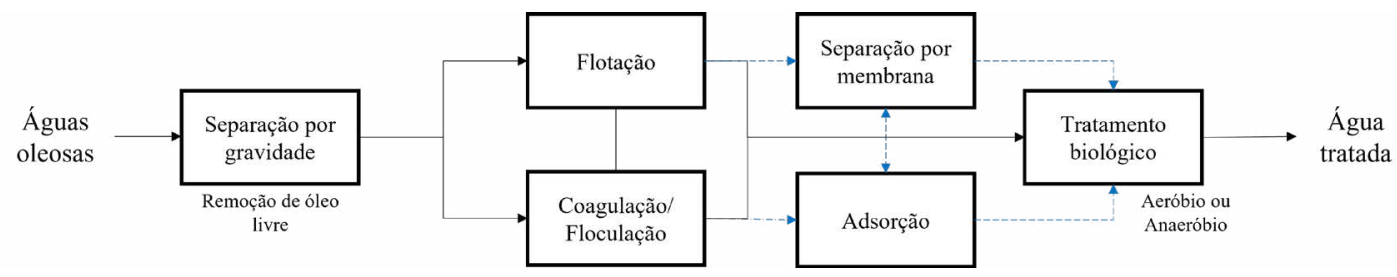

Figura 2 - Fluxograma simplificado de etapas

convencionais usadas em tratamento de águas oleosas. 
O óleo encontrado nos efluentes pode ser classificado de diferentes formas quanto ao tamanho das gotículas: flutuante ou livre (gotículas com diâmetro superior a $150 \mu \mathrm{m})$, disperso (20-150 $\mu \mathrm{m})$, emulsionado $(s 20 \mu \mathrm{m})$ e solúvel $(\leq 5 \mu \mathrm{m})$ [17]. A eficiência dos tratamentos depende da natureza do óleo e do tamanho das gotículas presentes.

\subsection{Processos físicos e químicos}

Entre as técnicas físico-químicas, destacam-se a separação por gravidade, flotação, coagulação/floculação, separação por membranas e adsorção [17].

A separação por gravidade tira vantagem da força gravítica, usando equipamentos que devem permitir tempos de retenção suficientes para que ocorra a formação de duas fases distintas. É o tratamento mais simples e é aplicado em etapas primárias, com o intuito de remover o óleo livre e os sólidos suspensos. Contudo, é pouco eficiente para separar óleo disperso, emulsionado ou solúvel $[4,17]$. Similarmente, a flotação também parte do princípio da formação de duas camadas distintas e conta com o auxílio de alimentação de ar no reator para arrastar as gotículas de óleo $[4,17]$, o que resulta num processo mais eficiente que a separação por ação da força gravítica.

O processo de coagulação/floculação pode ser induzido por métodos químicos ou eletrolíticos (eletrocoagulação) e baseia-se na formação de agregados de óleo [4,17]. A coagulação química inicia-se com a adição de um agente coagulante [4], que permite a formação de flocos. 0 processo de eletrocoagulação, por sua vez, utiliza passagem de corrente elétrica externa com o auxílio de dois elétrodos, sendo comum a utilização de cátodos de ferro e ânodos de alumínio. 0 processo de eletrocoagulação é bastante utilizado quando o efluente oleoso está emulsionado, uma vez que o processo de eletrólise conduz à quebra da emulsão [5]. As etapas de um processo de eletrocoagulação envolvem a (i) formação do agente coagulante pela oxidação eletrolítica do elétrodo de sacrifício, (ii) destabilização da fase dispersa resultando na quebra da emulsão e (iii) agregação da fase destabilizada para formar agregados (flóculos) de óleo $[5,17]$.

Os processos de separação por membrana estão entre os mais sofisticados e conduzem a um efluente de maior qualidade após o tratamento [17]. As vantagens da separação por membrana incluem uma elevada taxa de remoção do óleo (remoção de partículas na escala de $\approx$ 1-10 $\mu \mathrm{m}$ [20]), fácil operação, ausência de adição de agentes químicos e baixo consumo de energia. 0 principal desafio deste processo reside na acumulação de incrustantes [18], limitando a vida útil e a eficiência da membrana. 0 material da membrana e as condições de operação devem ser judiciosamente escolhidas com vista à rejeição adequada da fase oleosa. A eficiência de separação de uma membrana depende exclusivamente da membrana em si e é definida pelo coeficiente de rejeição de óleo (Ro), calculado pela diferença de concentração de óleo na alimentação e no permeado, a dividir pela concentração na alimentação $[4,17,18]$. Os processos de separação por membrana dependem de fatores como a pressão, a concentração de óleo, a temperatura e o tamanho do poro da membrana. Consoante estes fatores, podem ser designados por micro-, nano-, ou ultrafiltração, ou ainda, osmose inversa $[4,17]$. As membranas podem ser feitas de vários materiais, sendo os mais aplicados os poliméricos ou os cerâmicos [18].

A adsorção também é aplicada ao tratamento de águas oleosas e baseia-se na atração entre a superfície externa do adsorvente e do adsorbato (óleo) [17]. Adsorventes eficientes devem ser bastante lipofílicos, facilitando a retenção de óleo nos poros; características como alta capacidade e velocidade de retenção, durabilidade, reciclabilidade, possibilidade de recuperar o óleo adsorvido e elevada área superficial, são altamente desejadas [5,17]. 0 processo de adsorção é vantajoso porque não implica a adição de outros agentes químicos e permite atingir remoções elevadas de carência química de oxigénio (CQO). Contudo, o poluente é apenas transferido de uma fase aquosa para uma fase sólida, sendo preciso lidar adequadamente com o resíduo agregado ao adsorvente [21].

No caso específico de dois efluentes provenientes de uma refinaria de óleo vegetal, Pintor et al. [9] otimizaram um processo de separação por ação da força gravítica para remover óleo, gordura e CQO. Uma amostra foi recolhida no tanque de homogeneização (TH) da unidade de tratamento de efluentes de uma indústria e outra amostra recolhida após a etapa de remoção de ceras (RC). Com 24 h de tratamento foram removidos mais de $90 \%$ dos óleos e gorduras, em ambas as amostras, enquanto a remoção de CQO foi de $58 \%$ para a amostra TH e de $90 \%$ para a RC.

Através da simulação de uma água oleosa emulsionada (água da torneira, emulsificante (Tween 80) e gasóleo), Hoseini et al. [22] analisaram a eficiência da combinação de um processo de coagulação com flotação. Os ensaios experimentais foram conduzidos num equipamento de flotação, onde foram introduzidos a emulsão e o agente coagulante, com admissão de ar após 3 min de agitação a 600 rpm. A remoção máxima (93\%) de hidrocarbonetos de petróleo obteve-se usando sulfato de alumínio como agente coagulante, com uma concentração de $50 \mathrm{mg} \mathrm{L}^{-1}$, pH 4, 10 min de flotação, 1000 rpm de velocidade de agitação e um caudal de ar de 4,5 L min $^{-1}$.

Um processo de eletrocoagulação foi utilizado por Safari et al. [23] para reduzir a CQO e a quantidade de diesel presente num efluente aquoso simulado, 
com diferentes concentrações de diesel. Um máximo de remoção de 99,1\% de CQO e de 98,8\% de diesel (para uma concentração inicial de diesel de 3500 $\mathrm{mg} \mathrm{L}^{-1}$ ) foi obtido nas condições de pH 7, com 40 min de eletrocoagulação, a uma voltagem de 10,5 V, usando $\mathrm{NaCl}$ como eletrólito de suporte $\left(0,5 \mathrm{~g} \mathrm{~L}^{-1}\right)$, e elétrodos de alumínio. Nessas condições, produziram-se $1995 \mathrm{mg} \mathrm{L}^{-1}$ de lamas com um consumo energético de $6,47 \mathrm{kWh} \mathrm{m}^{3}$. Entretanto, ao aumentar a concentração inicial de diesel para $11000 \mathrm{mg} \mathrm{L}^{-1}$, a eficiência do processo diminui consideravelmente, para remoções de CQO e diesel de 49,1 e 45,5\%, respetivamente, acarretando um aumento na produção de lamas e no consumo energético do processo.

Membranas utilizando um compósito de $\mathrm{TiO}_{2} /$ $\mathrm{CO}_{3} \mathrm{O}_{4} /$ óxido de grafeno foram preparadas por Bao et al. [24] para serem utilizadas na separação de efluentes emulsionados simulados. Os efluentes foram preparados misturando diferentes óleos (tolueno, clorofórmio, hexano, gasolina e clorobenzeno) com água e um emulsificante. Efluentes emulsionados contaminados foram simulados através da adição de um corante (Vermelho do Congo). A membrana produzida apresenta características de super-moIhabilidade, bem como propriedades fotocatalíticas. A membrana demonstrou capacidade para segregar uma variedade de emulsões de óleo-água com eficiências superiores a 99,5\% por 10 ciclos. Além disso, em simultâneo com a quebra da emulsão, foi testada a degradação fotocatalítica com luz solar simulada do corante Vermelho do Congo, atingindo-se remoções de $82 \%$ do corante (concentração inicial de $10 \mathrm{mg} \mathrm{L}^{-1}$ ).

Chen et al. [6] usaram a separação por membranas combinada com um processo eletrolítico para desemulsificar um efluente oleoso (água oleosa contendo fluido de corte) e recuperar e reutilizar a fase oleosa. 0 processo permitiu aumentar o diâmetro das gotículas de emulsão de 7 a $55 \mu \mathrm{m}$, possibilitando uma redução de CQO de 87,9\% e uma recuperação de $5173 \mathrm{mg} \mathrm{L}^{-1} \mathrm{de}$ óleo (ciclos de 90 min a $10 \mathrm{~V}$, distância entre elétrodos de $10 \mathrm{~mm}$, membrana com poros de $5 \mu \mathrm{m}$, e caudal de $0,5 \mathrm{~L} \mathrm{~min}-1)$. A principal vantagem do processo proposto é a recuperação da fase oleosa.

Usando materiais de carbono modificados química, térmica e hidrotermicamente, Diaz de Tuesta et al. [25] estudaram a adsorção de um poluente lipofílico, o corante Sudan IV (S-IV) numa água oleosa simulada. O tratamento resultou na completa remoção de S-IV (concentração inicial de $500 \mathrm{mg} \mathrm{L}^{-1}$ ) em $8 \mathrm{~h} \mathrm{(pH}$ natural, $\left.50^{\circ} \mathrm{C}, \mathrm{C}_{\text {adsorvente }}=2,5 \mathrm{~g} \mathrm{~L}^{-1}\right)$. Os principais fatores que governam a capacidade de adsorção foram a lipofilicidade do material, a ausência de grupos oxigenados na sua superfície e a elevada área superficial. A presença da água não impediu a adsorção do S-IV. Contudo, a presença de emulsificantes dificulta o processo de adsorção e requer, previamente, a quebra da emulsão. 0 tratamento proposto permitiu remover o poluente lipofílico dissolvido na fase oleosa, permitindo a recuperação e reutilização dessa fase.

Os processos físico-químicos, como os descritos, são sobretudo processos de separação, i.e., destes processos resultam dois efluentes, um mais limpo e o outro mais concentrado em teor de gorduras e óleos. 0 concentrado ainda requer um tratamento ou destino adequados, pelo que é ainda necessário considerar algum processo adicional de degradação.

\subsection{Processos biológicos}

Os processos biológicos podem ser conduzidos de forma aeróbia ou anaeróbia, e podem ser utilizados diversos microrganismos (fungos, bactérias, algas) $[2,16]$, sendo quatro as fontes mais comuns: os que ocorrem naturalmente neste tipo de efluentes, microrganismos comerciais, grupos específicos de microrganismos, ou lodo de esgoto aclimatado [16]. 0 processo usando lamas ativadas é um dos mais aplicados [4,16]. Neste processo, o efluente contaminado entra em contacto com microrganismos num tanque arejado. Os microrganismos usam o material orgânico presente no efluente para os seus processos metabólicos, levando à degradação da matéria orgânica [4].

Supõe-se que o mecanismo pelo qual os hidrocarbonetos são removidos por lamas ativadas inclui um passo de floculação, uma vez que a lama ativada tem a capacidade de adsorver compostos solúveis e insolúveis, formando agregados [16]. A degradação de componentes oleosos menos complexos, como alcenos lineares, é mais fácil do que a de componentes com cadeias mais complexas, o que acarreta a fixação de componentes menos biodegradáveis aos microrganismos, permanecendo no tanque de aeração. Esses conjugados são removidos juntamente com o excesso de lama ativada ao longo do tempo e devem ser devidamente descartados devido ao alto poder poluente [16]. Para concentrações muito altas de poluentes, a utilização de técnicas anaeróbicas é uma alternativa mais eficiente em termos de custos [16].

A integração de um reator anaeróbico (upflow anaerobic sludge blanket - UASB) com dois reatores do tipo biological aerated filter (BAF) em série no tratamento de um efluente oleoso contendo alto teor de compostos recalcitrantes e baixos teores de nitrogénio e fósforo foi investigada por Zou [10]. 0 ensaio foi seguido por 180 dias, variando o tempo de retenção do efluente em cada uma das etapas, levando a altas remoções de CQO (90,2\%), nitrogénio amoniacal (90,8\%), conteúdo de óleos (86,5\%) e hidrocarbonetos aromáticos $(89,4 \%)$. 0 reator UASB atua no aumento da biodegradabilidade do efluente (a razão $\mathrm{CBO}_{5} / \mathrm{CQO}$ aumenta de 0,21 para 0,45 ), enquanto o primeiro reator 
BAF tem um papel crucial na diminuição da CQO dos óleos e dos hidrocarbonetos aromáticos.

0 tratamento de águas residuais provenientes de restaurantes com um elevado teor de óleos foi estudado por Gao et al. [26]. Os microrganismos usados no estudo foram isolados de lamas oleosas, recolhidas num esgoto próximo de uma cantina universitária, e permitiram degradar $>85 \%$ de óleo (conteúdo inicial entre $2-4 \%$ v/v) em 30 h, a pH 7-10 e à temperatura de $25-35^{\circ} \mathrm{C}$. No entanto, o aumento da concentração inicial de óleos vegetais afeta a capacidade de degradação desses microrganismos, baixando as remoções para menos de $45 \%$ em concentrações iniciais de óleo de 10\% v/v.

A bactéria Aeribacillus pallidus presente num efluente de uma refinaria de petróleo foi isolada por Ktata et al. [11] e utilizada na produção de uma lipase alcalina, e testada no tratamento de uma água oleosa proveniente de uma indústria produtora de atum enlatado. A lipase demonstrou bastante resistência à temperatura, $\mathrm{pH}$, e à presença de detergentes $\mathrm{e}$ solventes orgânicos. Após $1 \mathrm{~h}$ de tratamento, foi observada uma redução de $83 \%$ de CQ0, $85 \%$ de $\mathrm{CBO}_{5}$ e de $96,1 \%$ de lípidos (concentrações iniciais de $2178 \mathrm{mg} \mathrm{L}^{-1}, 910 \mathrm{mg} \mathrm{L}^{-1}$ e $641 \mathrm{mg} \mathrm{L}^{-1}$ para CQO, $\mathrm{CBO}_{5} \mathrm{e}$ lípidos, respetivamente).

Os processos de tratamento biológicos podem apresentar algumas vantagens face aos processos físico-químicos. No entanto, a presença de óleos é um desafio para o uso de microrganismos, uma vez que o óleo pode formar uma camada isolante, impedindo as trocas de oxigénio e de nutrientes, reduzindo a eficiência do processo [17]. Além disso, são estratégias que por vezes requerem o acondicionamento ou adaptação dos microrganismos e, portanto, menos versáteis. Adicionalmente, levam também à degradação indiferenciada da fase oleosa, e no caso de fases oleosas de alto valor acrescentado, há uma perda económica associada.

\section{Processos avançados de oxidação (AOPs)}

Os AOPs são processos alternativos de tratamento de águas que permitem altas conversões de poluentes orgânicos, levando à formação de água e de dióxido de carbono [27]. Existem diversos AOPs, como o processo de Fenton, oxidação catalítica com peróxido de hidrogénio, ozonização, eletro-oxidação, fotólise e fotocatálise, que podem ser escolhidos de acordo com as características do efluente a ser tratado, as condições locais de tratamento, os custos e a instalação de equipamentos [28]. A maioria dos AOPs são baseados na formação seletiva do radical hidroxilo (HO·) que é conhecido pelo seu elevado poder oxidante (potencial de redução padrão entre 2,8 e 2,0 V a pH 0 e 14, respetivamente [29]) e pela sua capacidade de oxidar não-seletivamente uma série de compostos orgânicos que costumam ser recalcitrantes em tratamentos físico-químicos e biológicos [30]. Os AOPs mostraram-se bastante eficientes para tratar uma variedade de efluentes, incluindo águas oleosas [31-35], e permitem abordagens para a recuperação da fase oleosa [3,36-38].

Águas oleosas simuladas por Jimenéz et al. [33] foram processadas através da combinação de um pré-tratamento por um sistema de coagulação/floculação/flotação, seguido por Fenton e foto-Fenton. As águas foram simuladas usando uma mistura de azeite, gasolina e fenol, resultando numa CQO de 342,5 mg L-1. Após o pré-tratamento, ainda havia a presença de óleos, gorduras, e fenol residuais. 0 tratamento subsequente por foto-Fenton levou a uma degradação de $75 \%$ do conteúdo residual de óleo e gordura e mais de $90 \%$ do fenol em apenas 3 h de reação ( $\mathrm{pH} 3$, irradiação de $250 \mathrm{~W} \mathrm{~m}^{-2}$, temperatura $30-35^{\circ} \mathrm{C}$, concentração de peróxido de hidrogénio de $600 \mathrm{mg} \mathrm{L}^{-1}$ ), enquanto o tratamento por Fenton não permitiu atingir os mesmos resultados.

A ozonização foi utilizada por Uma e Gandhimathi [34] como pré-tratamento para um efluente oleoso proveniente de um porão de navio, caracterizado por conter hidrocarbonetos de petróleo, sais, surfactantes, óleos, solventes de limpeza e metais. 0 pré-tratamento com ozono permitiu aumentar o índice de biodegradabilidade $\left(\mathrm{CBO}_{5} / \mathrm{CQO}\right)$ de 0,36 para 0,52 (75 min de tratamento, $\mathrm{pH} 6$, e dose de ozono de $2 \mathrm{~g} \mathrm{~L}^{-1}$ ). A combinação de ozonização e reator anaeróbio levou a uma remoção de 92\% de CQO. Em comparação, sem o pré-tratamento com ozono, houve apenas 68\% de remoção de CQO.

Águas provenientes de poços de extração off-shore foram tratadas por Liu et al. [35] por ozonização fotocatalítica, usando como catalisador uma matriz de nanotubos de $\mathrm{TiO}_{2}$ e como fonte de irradiação um díodo ultravioleta (UV-LED). Foi possível eliminar completamente os hidrocarbonetos aromáticos presentes na solução (30 min de tratamento, dose de ozono de $15 \mathrm{mg}_{0_{3}} \mathrm{~L}_{\mathrm{O}_{2}}{ }^{-1}$ e $\left.\mathrm{C}_{\text {catalisador }}=0,2 \mathrm{~g} \mathrm{~L}^{-1}\right)$, sendo o processo de ozonização o principal responsável por essa diminuição. A fotólise por si só não permitiu a degradação dos hidrocarbonetos aromáticos. A biodegradabilidade do efluente $\left(\mathrm{CBO}_{28} / \mathrm{CQO}\right)$ aumentou consideravelmente, de 0,45 para 0,85.

Foram também reportados sistemas baseados em AOPs que permitem o tratamento da fase oleosa, a maior parte focados em remover compostos de enxofre e/ou nitrogénio, dado o interesse industrial em remover essa classe de compostos de combustíveis líquidos [39]. Diaz de Tuesta et al. [3] simularam uma água oleosa contaminada com um poluente lipofílico (2-nitrofenol, 2-NP) e estudaram o tratamento deste 
efluente através de oxidação seletiva com peróxido de hidrogénio, com o objetivo de tratar a fase oleosa. Foram utilizados nanotubos de carbono com diferentes graus de afinidades pelas fases óleo-água (materiais anfifílicos, um material completamente hidrofílico e outro material completamente lipofílico). Na presença dos materiais anfifílicos, a remoção do 2-NP aumentou consideravelmente se comparada com a observada pela presença dos materiais completamente hidrofílico ou lipofílico. A melhoria na remoção seletiva do poluente foi atribuída à capacidade dos materiais anfifílicos estabilizarem emulsões de Pickering, bem como promoverem a decomposição seletiva do $\mathrm{H}_{2} \mathrm{O}_{2}$ em radicais hidroxilo. A formação da emulsão, conforme representado no esquema proposto na Figura 3, permitiu um contacto mais próximo entre poluente e agente oxidante, aumentando a eficiência do processo. Foi possível uma remoção de $76,7 \%$ do 2-NP inicialmente presente $\left(50{ }^{\circ} \mathrm{C}, \mathrm{pH} 3, \mathrm{C}_{\text {catalisador }}=\right.$ $0,25 \mathrm{~g} \mathrm{~L}^{-1}, \mathrm{C}_{2-\mathrm{NP}}=0,5 \mathrm{~g} \mathrm{~L}^{-1}, \mathrm{C}_{\mathrm{H}_{2} \mathrm{O}_{2}}$ estequiométrica para conversão completa do 2-NP) após 24 h de reação. 0 processo permite a eliminação de um poluente lipofílico dissolvido numa fase oleosa sem prejudicar a qualidade desta, possibilitando a sua recuperação. Mais recentemente, os mesmos autores avaliaram o mesmo tratamento procurando valorizar um precursor mais barato para a produção de materiais à base de carbono [40]. o composto maturado, procedente do tratamento mecânico e biológico de resíduos sólidos municipais indiferenciados, foi usado como precursor. Remoções superiores a $80 \%$ foram possíveis $(\mathrm{pH} 3$, $\mathrm{C}_{\text {catalisador }}=2,5 \mathrm{~g} \mathrm{~L}^{-1}$, quantidade estequiométrica de $\mathrm{H}_{2} \mathrm{O}_{2}$ para conversão completa dos poluentes, $24 \mathrm{~h}$ de reação) tanto para o 2-NP $\left(0,5 \mathrm{~g} \mathrm{~L}^{-1}\right)$ como para 0 4-nitrofenol (5,0 $\left.\mathrm{g} \mathrm{L}^{-1}\right)$ em misturas ciclo-hexano/água.
Outros autores reportaram também sistemas similares ao proposto na Figura 3. Oliveira et al. [36] sintetizaram nanotubos de carbono com diferentes graus de anfifilicidade e testaram a sua utilização na remoção de S-IV, tiofeno, dibenzotiofeno (DBT) e quinolina (QN) em meio bifásico, levando a remoções de 80, 67, 82 e $100 \%$ (S-IV, tiofeno, DBT e QN, respetivamente) em $1 \mathrm{~h}$ de reação. Teixeira et al. [37] produziram nanotubos de carbono sobre $\mathrm{Al}_{2} \mathrm{O}_{3}$ contendo Fe/Mo e aplicaram-nos num meio bifásico com $\mathrm{H}_{2} \mathrm{O}_{2}$ para remover DBT e QN de uma fase oleosa, tendo sido observada a sua completa oxidação em 200 e 30 min de reação, respetivamente. Oliveira et al. [41] usaram compósitos de SiC na oxidação bifásica de compostos sulfurados numa amostra de diesel, permitindo uma remoção de 57\% desses compostos. Santos et al. [38] testaram compósitos de nanotubos de carbono/nanotubos de titânio para a oxidação em meio bifásico do amarelo de metilo (MY) e do álcool benzílico com $\mathrm{H}_{2} \mathrm{O}_{2}$, atingindo $100 \%$ de remoção de $30 \mathrm{mg} \mathrm{L}^{-1}$ de $M Y$ em 60 min de reação e mais de $90 \%$ de álcool benzílico em 3 h de reação. Mambrini et al. [42] produziram nanotubos de carbono sobre óxidos de Fe/Mo para oxidação em meio bifásico de DBT e QN, usando $\mathrm{H}_{2} \mathrm{O}_{2}$, levando a 63 e 100\% de conversão, respetivamente. Sun et al. [43] estudaram a aplicação de um catalisador de óxido de tungsténio na oxidação bifásica para remover DBT (100\%), mantendo a atividade catalítica até 5 ciclos. Yue et al. [44] imobilizaram ácido fosfotúngstico sobre sílica para a remoção em meio bifásico de DBT, benzotiofeno ou 4,6-dimetildibenzotiofeno, atingindo remoções de 100, 91 e $83 \%$, respetivamente. Jatav e Srivastava [45] sintetizaram óxido misto de CeAl e usaram-no na oxidação bifásica para remoção de DBT, atingindo $100 \%$ de remoção. a)

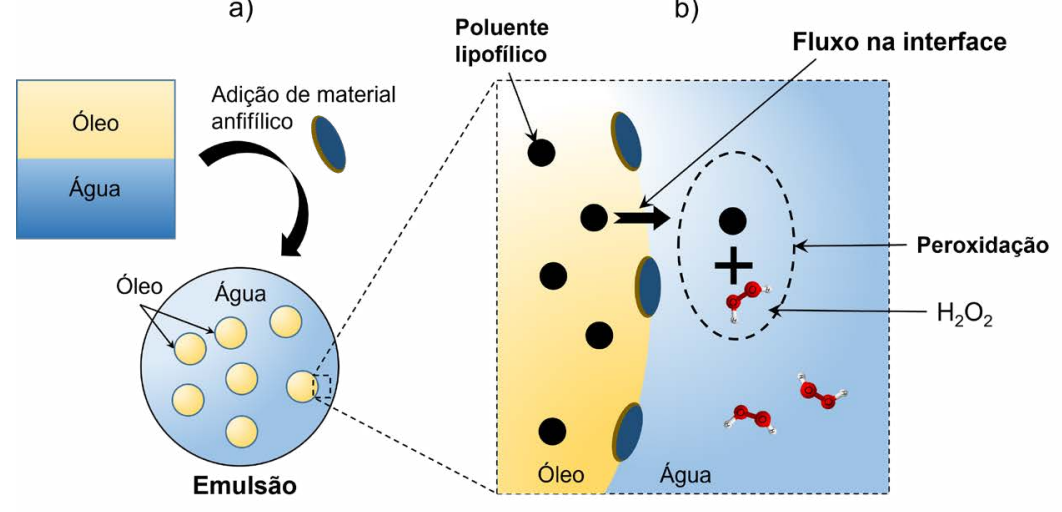

Figura 3 - Esquema proposto para tratamento de águas oleosas por processos de oxidação avançados usando materiais anfifílicos simultaneamente como catalisadores e estabilizadores de emulsão simultaneamente como catalisadores e estabilizado
de Pickering. Adaptado de Diaz de Tuesta et al. [3]. c)

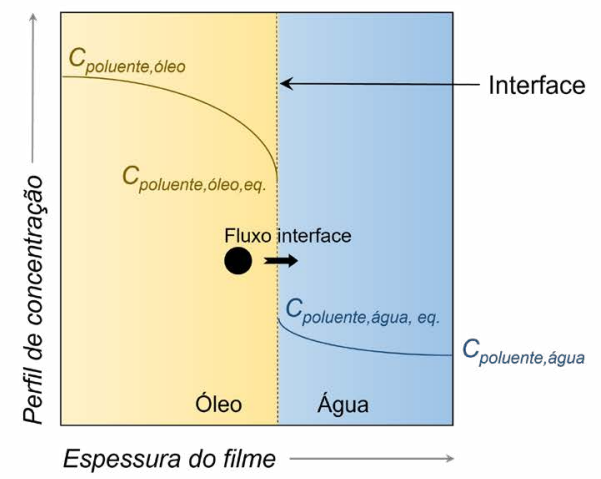


Conclui-se que os AOPs são bastante eficientes no tratamento de águas oleosas, podendo ser aplicados com diversos objetivos: degradação do óleo presente no efluente, aumento da biodegradabilidade da água oleosa para um subsequente tratamento biológico, ou tratamento simultâneo da fase oleosa e da fase aquosa. Assim, são tecnologias versáteis e que reúnem uma série de vantagens face às tecnologias tradicionais, como seja a degradação sem geração de um novo efluente concentrado. Além disso, podem converter componentes orgânicos com toxicidade para os microrganismos utilizados nos tratamentos biológicos. É de enfatizar a possibilidade de recuperar uma fase oleosa de elevado valor acrescentado.

\section{Considerações finais}

As águas oleosas são efluentes com características muito variáveis dependendo da fonte emissora, sendo de difícil tratamento devido à presença de outros poluentes orgânicos e inorgânicos, para além do próprio óleo/gordura. Os métodos atuais de tratamento têm dificuldade em atingir parâmetros aceitáveis para descarga em recursos hídricos, sendo necessário encontrar alternativas viáveis que permitam tratar adequadamente este tipo de efluentes. Entre as várias possibilidades, as alternativas que permitam recuperar simultaneamente o efluente aquoso e a fase oleosa têm sido alvo de maior atenção, permitindo somar benefícios económicos aos ambientais. No entanto, existem poucos trabalhos na literatura focados no tratamento da fase oleosa. Menos ainda são os que propõem soluções integradas para tratar a água e recuperar o óleo. Os AOPs emergem de forma promissora já que permitem: (i) degradar seletivamente compostos prejudiciais à qualidade da água e da fase oleosa; (ii) recuperar e reutilizar a fase oleosa com valor económico acrescentado; e (iii) tratar a fase aquosa, atingindo os limites estabelecidos na legislação para os parâmetros de água de consumo humano.

\section{Agradecimentos}

Os autores agradecem à Fundação para a Ciência e a Tecnologia (FCT, Portugal) pelo apoio financeiro ao CIMO (UIDB/00690/2020) e ao Laboratório associado LSRE-LCM (referência UIDB/50020/2020) através de fundos nacionais FCT/MCTES. Fernanda F. Roman agradece à Fundação para a Ciência e a Tecnologia e ao Fundo Social Europeu a bolsa de doutoramento com referência SFRH/BD/143224/2019.

\section{Referências}

[1] L. Hui, W. Yan, W. Juan, L. Zhongming, Recent Innov. Chem. Eng. 2015, 7, 17-24. DOI: $10.2174 / 2211334707666140415222545$.

[2] S. Jamaly, A. Giwa, S. W. Hasan, J Environ. Sci. 2015, 37, 15-30. DOI: 10.1016/j.jes.2015.04.011.

[3] J. L. Diaz de Tuesta, B. F. Machado, P. Serp, A. M. T. Silva, J. L. Faria, H. T. Gomes, Catal. Today 2020, 356, 205-215. DOI: 10.1016/j. cattod.2019.07.012.

[4] S. Varjani, R. Joshi, V. K. Srivastava, H. H. Ngo, W. Guo, Environ. Sci. Pollut. Res. 2020, 27, 27172-27180. DOI: 10.1007/s11356-019-04725-x.

[5] C. An, G. Huang, Y. Yao, S. Zhao, Sci. Total Environ. 2017, 579, 537-556. DOI: 10.1016/j.scitotenv.2016.11.062

[6] P. Chen, D. Yin, P. Song, Y. Liu, L. Cai, H. Wang, L. Zhang, J. Clean. Prod. 2020 244, 118698. DOI: 10.1016/j.jclepro.2019.118698.

[7] W. Pitakpoolsil, M. Hunsom, J. Taiwan, Inst. Chem. Eng. 2013, 44, 963-971. DOI: 10.1016/j.jtice.2013.02.009.

[8] J. X. Kang, L. Lu, W. Zhan, B. Li, D. S. Li, Y. Z. Ren, D. Q. Liu, J. Hazard. Mat. 2011, 186, 849-854. DOI: 10.1016/j.jhazmat.2010.11.075.

[9] A. M. A. Pintor, V. J. P. Vilar, C. M. S. Botelho, R. A. R. Boaventura, Clean Technol. Environ. Policy 2014, 16, 1725-1734. DOI: 10.1007/s10098-0140754-3.

[10] X. L. Zou, Environ. Technol. 2015, 36, 2381-2389. DOI $10.1080 / 09593330.2015 .1030346$.

[11] A. Ktata, N. Krayem, A. Aloulou, S. Bezzine, A. Sayari, M. Chamkha, A. Karray, J. Biochem. 2020, 167, 89-99. DOI: 10.1093/jb/mvz083.

[12] F. Ma, J. B. Guo, L. J. Zhao, C. C. Chang, D. Cui, Bioresour. Technol. 2009, 100, 597-602. DOl: 10.1016/j.biortech.2008.06.066

[13] C. E. Santo, V. J. P. Vilar, A. Bhatnagar, E. Kumar, C. M. S. Botelho, R. A. R. Boaventura, Desalin. Water Treat. 2013, 51, 6641-6654. DOI: 10.1080/19443994.2013.792141.
[14] Decreto-Lei n. ${ }^{\circ}$ 236/98 de 1 de agosto, Diário da República n. ${ }^{\circ}$ 176/98 - 1. ${ }^{\text {a }}$ Série. Ministério do Ambiente. Lisboa. dre.pt/application/conteudo/430457.

[15] P. E. Ndimele, A. O. Saba, D. O. Ojo, C. C. Ndimele, M. A. Anetekhai, E. S. Erondu, Remediation of Crude Oil Spillage, in P. E. Ndimele (Ed.). The Political Ecology of Oil and Gas Activities in the Nigerian Aquatic Ecosystem, Candice Janco, Chennai, Índia, 2018

[16] A. Fakhru'I-Razi, A. Pendashteh, L. C. Abdullah, D. R. Biak, S. S. Madaeni, Z. Z. Abidin, J. Hazard, Mat. 2009, 170, 530-551. DOI: 10.1016/j. jhazmat.2009.05.044

[17] P. Kundu, I. M. Mishra, Rev. Chem. Eng. 2018, 35, 73-108. DOI: 10.1515/ revce-2017-0025

[18] M. Padaki, R. S. Murali, M. S. Abdullah, N. Misdan, A. Moslehyani, M. A. Kassim, N. Hilal, A. F. Ismail, Desalination 2015, 357, 197-207. DOI: 10.1016/j. desal.2014.11.023.

[19] Decreto-Lei n. $.^{\circ} 119 / 2019$ de 21 de agosto, Diário da República n. 159/2019, 1. ${ }^{a}$ série. Presidência do Conselho de Ministros. Lisboa. dre.pt/application/ conteudo/124097549.

[20] H. J. Tanudjaja, C. A. Hejase, V. V. Tarabara, A. G. Fane, J. W. Chew, Water. Res. 2019, 156, 347-365. DOI: 10.1016/j.watres.2019.03.021.

[21] S. Jimenez, M. M. Mico, M. Arnaldos, F. Medina, S. Contreras, Chemosphere 2018, 192, 186-208. DOI: 10.1016/j.chemosphere.2017.10.139.

[22] S. M. Hoseini, M. M. Salarirad, M. R. A. Moghaddam, Desalin. Water Treat. 2013, 53, 300-308. DOI: 10.1080/19443994.2013.846522.

[23] S. Safari, M. A. Aghdam, H. R. Kariminia, Int. J. Environ. Sci. Technol. 2015, 13, 231-242. DOI: 10.1007/s13762-015-0863-5.

[24] Z. Bao, D. Chen, N. Li, Q. Xu, H. Li, J. He, J. Lu, J. Membr. Sci. 2020, 598, 117804. DOI: 10.1016/j.memsci.2019.117804.

[25] J. L. Diaz de Tuesta, A. M. T. Silva, J. L. Faria, H. T. Gomes, Chem. Eng. J. 2018, 347, 963-971. DOI: 10.1016/j.cej.2018.04.105. 
[26] L. L. Gao, Y. C. Lu, J. L. Zhang, J. Li, J. D. Zhang, World J. Microbiol. Biotechnol. 2019, 35, 179. DOl: 10.1007/s11274-019-2760-4.

[27] R. Anjali, S. Shanthakumar, J. Environ. Manag. 2019, 246, 51-62. DOI: 10.1016/j.jenvman.2019.05.090.

[28] C. Teodosiu, A.-F. Gilca, G. Barjoveanu, S. Fiore, J. Clean. Prod. 2018, 197, 1210-1221. DOI: 10.1016/j.jclepro.2018.06.247.

[29] S. Navalon, M. Alvaro, H. Garcia, Appl. Catal. B-Environ. 2010, 99, 1-26. DOI: 10.1016/j.apcatb.2010.07.006.

[30] R. S. Ribeiro, A. M. T. Silva, J. L. Figueiredo, J. L. Faria, H. T. Gomes, Appl. Catal. B-Environ. 2016, 187, 428-460. DOl: 10.1016/j.apcatb.2016.01.033.

[31] J. Zhang, J. Li, R. Thring, L. Liu, Procedia Environ. Sci. 2013, 18, 686-693. DOl: 10.1016/j.proenv.2013.04.093.

[32] K. Sivagami, D. Anand, G. Divyapriya, I. Nambi, Ultrason. Sonochem. 2019, 51, 340-349. DOI: 10.1016/j.ultsonch.2018.09.007.

[33] S. Jiménez, M. M. Micó, M. Arnaldos, E. Ferrero, J. J. Malfeito, F. Medina, S. Contreras, Chemosphere 2017, 168, 309-317. DOI: 10.1016/j. chemosphere.2016.10.055.

[34] V. Uma, R. Gandhimathi, J. Water Process. Eng. 2020, 36, 101275. DOI: 10.1016/j.jwpe.2020.101275.

[35] B. Liu, B. Chen, B. Zhang, X. Song, G. Zeng, K. Lee, J. Hazard. Mat. 2021, 402, 123456. DOI: 10.1016/j.j.hazmat.2020.123456.

[36] A. A. S. Oliveira, I. F. Teixeira, T. Christofani, J. C. Tristão, I. R. Guimarães, F. C. C. Moura, Appl. Catal. B-Environ. 2014, 144, 144-151. DOI: 10.1016/j. apcatb.2013.07.015.
[37] I. F. Teixeira, A. A. d. S. Oliveira, T. Christofani, F. C. C. Moura, J. Mat. Chem. A 2013, 1, 10203-10208. DOI: 10.1039/C3TA11535F.

[38] S. R. A. Santos, I. S. Jardim, H. A. Bicalho, I. Binatti, E. M. B. Sousa, A. M. Peres, R. R. Resende, E. Lorencon, J. Colloid Interface Sci. 2016, 483, 211-219. DOI: 10.1016/j.jcis.2016.08.025

[39] P. Sikarwar, V. Gosu, V. Subbaramaiah, Rev. Chem. Eng. 2019, 35, 669-705. DOI: 10.1515/revce-2017-0082.

[40] J. L. Diaz de Tuesta, G. F. Pantuzza, A. M. T. Silva, P. Praça, J. L. Faria, H. T. Gomes, Catalysts 2020, 10, 1243. DOI: 10.3390/catal10111243.

[41] A. A. S. Oliveira, T. Christofani, I. F. Teixeira, J. D. Ardisson, F. C. C. Moura, New J. Chem. 2015, 39, 5445-5452. DOl: 10.1039/C5NJ00593K.

[42] R. V. Mambrini, C. Z. Maia, J. D. Ardisson, P. P. de Souza, F. C. C. Moura, New J. Chem. 2017, 41, 142-150. DOI: 10.1039/C6NJ02718K.

[43] H. Sun, P. Wu, J. He, M. Liu, L. Zhu, F. Zhu, G. Chen, M. He, W. Zhu, Pet. Sci. 2018, 15, 849-856. DOI: 10.1007/s12182-018-0265-7.

[44] D. Yue, J. Lei, Z. Lina, G. Zhenran, X. Du, J. Li, Catal. Lett. 2018, 148, 11001109. DOI: 10.1007/s10562-018-2317-4.

[45] S. Jatav, V. C. Srivastava, Pet. Sci. Technol. 2019, 37, 633-640. DOl: 10.1080/10916466.2018.1560323.

\section{Adrián M. T. Silva}

Laboratório de Processos de Separação e Reação-Laboratório de Catálise e Materiais (LSRE-LCM).

Faculdade de Engenharia, Universidade do Porto.

Licenciou-se em Engenharia Química pela Faculdade de Ciências e Tecnologia da Universidade de Coimbra. Recebeu o título de Doutor pela mesma Faculdade em 2005. É Professor na Faculdade de Engenharia da Universidade do Porto (FEUP). A sua experiência está focada na preparação, caracterização e aplicação de materiais nano e macroestruturados para separação e engenharia de reação. adrian@fe.up.pt ORCID.org/0000-0001-8551-6353

\section{$>$}

\section{Fernanda F. Roman}

Centro de Investigação de Montanha (CIMO).

Instituto Politécnico de Bragança.

Laboratório de Processos de Separa-

ção e Reação-Laboratório de Catálise e Materiais (LSRE-LCM).

Faculdade de Engenharia, Universidade do Porto.

Licenciou-se em Engenharia Química na Universidade Tecnológica Federal do Paraná, no Brasil. Atualmente, é estudante de doutoramento em Engenharia Química na Faculdade de Engenharia da Universidade do Porto. Desenvolve seu trabalho de pesquisa no Centro de Investigação de Montanha, Instituto Politécnico de Bragança.

roman@ipb.pt

ORCID.org/0000-0001-5360-5298

\section{${ }^{*}$ Helder T. Gomes}

Centro de Investigação de Montanha (CIMO).

Instituto Politécnico de Bragança.

Licenciou-se em Engenharia Química pela Faculdade de Engenharia da Universidade do Porto (FEUP). Em 2002, obteve o grau de Doutor também pela FEUP. Desde 2001 atua como professor no Instituto Politécnico de Bragança (IPB). Os seus principais interesses de investigação estão focados na síntese e caracterização de materiais heterogéneos à base de carbono para aplicações ambientais, biomédicas e de bioenergia. htgomes@ipb.pt

ORCID.org/0000-0001-6898-2408

\section{$>$}

\section{Joaquim L. Faria}

Laboratório de Processos de Separação e Reação-Laboratório de Catálise e Materiais (LSRE-LCM).

Faculdade de Engenharia, Universidade do Porto.

Doutorado em Química pelo Instituto Superior Técnico da Universidade Técnica de Lisboa (1993), com base nos trabalhos realizados no Max-Planck-Institut für Strahlenchemie, Alemanha. Em 1992, ingressou na Faculdade de Engenharia da Universidade do Porto, onde é agora Professor Associado com agregação em Engenharia Química e
Biológica. O seu domínio de investigação está centrado em catálise para aplicações de química ambiental, de energia e de síntese química.

jlfaria@fe.up.pt ORCID.org/0000-0002-6531-3978

\section{Jose L. Diaz de Tuesta}

Centro de Investigação de Montanha (CIMO).

Instituto Politécnico de Bragança.

É doutor em Engenharia Química pela Universidad Autónoma de Madrid. Desde 2016, é investigador no Centro de Investigação de Montanha, Instituto Politécnico de Bragança. Atualmente, estuda a valorização de resíduos sólidos para a produção de materiais de carbono para aplicações em adsorção ou catálise, principalmente no tratamento de águas residuais.

jl.diazdetuesta@ipb.pt ORCID.org/0000-0003-2408-087X 